\title{
An explicit formula for derivative polynomials of the tangent function
}

\author{
Feng Qi \\ Institute of Mathematics, \\ Henan Polytechnic University, China \\ College of Mathematics, \\ Inner Mongolia University for \\ Nationalities, China \\ Department of Mathematics, \\ College of Science, \\ Tianjin Polytechnic University, China \\ email: qifeng618@gmail.com, \\ qifeng618@gmail.com
}

\author{
Bai-Ni Guo \\ School of Mathematics and Informatics, \\ Henan Polytechnic University, China \\ email: bai.ni.guo@gmail.com, \\ bai.ni.guo@hotmail.com
}

\begin{abstract}
In the paper, the authors derive an explicit formula for derivative polynomials of the tangent function, deduce an explicit formula for tangent numbers, pose an open problem about obtaining an alternative and explicit formula for derivative polynomials of the tangent function, and recommend some papers closely related to derivative polynomials of other elementary and applicable functions.
\end{abstract}

\section{Introduction}

It is not difficult to see that if $f$ is a function whose derivative is a polynomial in $f$, that is, $f^{\prime}(x)=P_{1}(f(x))$ for some polynomial $P_{1}$, then all the higher order derivatives of $f$ are also polynomials in $f$, so we have a sequence of polynomials

2010 Mathematics Subject Classification: 26C99, 26A06, 26A09, 16A24, 33B10, 42A05 Key words and phrases: derivative polynomial; tangent function; explicit formula; tangent number; open problem 
$P_{n}$ defined by $f^{(n)}(x)=P_{n}(f(x))$ for $n \geq 0$. As usual, we call $P_{n}(u)$ the derivative polynomials of $f$. In fact, the polynomials $P_{n}$ are determined by

$$
P_{0}(u)=u, \quad P_{n+1}(u)=P_{n}^{\prime}(u) P_{1}(u), \quad n \in \mathbb{N} .
$$

For detailed information, please refer to [8, Section 2].

In 1945, Morley [10] observed that

$$
\begin{gathered}
(\tan x)^{\prime}=1+\tan ^{2} x, \quad(\tan x)^{\prime \prime}=2 \tan x+2 \tan ^{3} x, \\
(\tan x)^{\prime \prime \prime}=2+(2+2 \cdot 3) \tan ^{2} x+2 \cdot 3 \tan ^{4} x
\end{gathered}
$$

a term $a_{k} \tan ^{k} x$ in $(\tan x)^{(n)}$ gives $(\tan x)^{(n+1)} k a_{k} \tan ^{k-1} x+k a_{k} \tan ^{k+1} x$, and then concluded that the coefficient of $\tan ^{k-1} x$ in $(\tan x)^{(n+1)}$ is $(k-2) a_{k-2}+$ $k a_{k}$, with $a_{k-2}=0$ when $k \leq 1$, and $a_{k}=0$ when $k \geq n+2$.

In 1995, Hoffman [8, p. 25, (5)] obtained that the derivative polynomials $P_{n}$ for the tangent function $\tan x$ defined by

$$
\frac{d^{n}(\tan x)}{d x^{n}}=P_{n}(\tan x)
$$

for $n \geq 0$ are polynomials of degree $n+1$ and satisfy the recurrence relation

$$
P_{n+1}(u)=\sum_{k=0}^{n}\left(\begin{array}{l}
n \\
k
\end{array}\right) P_{k}(u) P_{n-k}(u)+\delta_{0 n},
$$

where

$$
P_{0}(u)=u, \quad P_{1}(u)=1+u^{2}, \quad \text { and } \quad \delta_{i j}= \begin{cases}0, & i \neq j \\ 1, & i=j .\end{cases}
$$

In $[1,9,12,26,27,32,36]$, there are some explicit formulas and recurrence relations for the $\mathrm{nth}$ derivatives of trigonometric functions and other elementary functions. In $[3,4,5,20,21,26,30,33]$, there are some inequalities for trigonometric functions and other elementary functions. Specially, there are some explicit formulas and many other results on the nth derivative of the tangent function $\tan x$ in $[11,14]$.

Motivated by those results in $[8,10]$ and other references mentioned above, we are interested in the question: can one find explicit formulas for coefficients $a_{k}$ of the derivative polynomials $P_{n}(u)$ for the tangent function $\tan \chi$ ?

The aim of this paper is to answer the above question. Our main results can be stated as the following theorem. 
Theorem 1 For $\mathrm{n} \geq 0$, the derivative polynomials $\mathrm{P}_{\mathrm{n}}(\mathrm{u})$ of the tangent function $\mathrm{u}=\tan \mathrm{x}$ can be explicitly computed by

$$
P_{n}(u)=\sum_{k=0}^{\frac{1}{2}\left[n+\frac{1-(-1)^{n}}{2}\right]} a_{n, n+1-2 k} u^{n+1-2 k}
$$

with

$$
a_{2 m-1,0}=(-1)^{m} \sum_{\ell=1}^{2 m}(-1)^{\ell} 2^{2 m-\ell}(\ell-1) ! S(2 m, \ell)
$$

for $m \geq 1$ and

$$
a_{n, n+1-2 k}=(-1)^{k-1} \sum_{\ell=n+1-2 k}^{n+1}(-1)^{n-\ell} 2^{n+1-\ell}(\ell-1) !\left(\begin{array}{c}
\ell \\
n+1-2 k
\end{array}\right) S(n+1, \ell)
$$

for $0 \leq \mathrm{k} \leq \frac{1}{2}\left[\mathrm{n}-\frac{1-(-1)^{\mathrm{n}}}{2}\right]$, where $\mathrm{S}(\mathrm{n}, \mathrm{k})$ for $\mathrm{n} \geq \mathrm{k} \geq 1$ stand for the Stirling numbers of the second kind which can be generated by

$$
\frac{\left(e^{x}-1\right)^{k}}{k !}=\sum_{n=k}^{\infty} S(n, k) \frac{x^{n}}{n !}, \quad k \in \mathbb{N} .
$$

In Section 3 of this paper, we will pose an open problem about obtaining an alternative and explicit formula

$a_{n, n-2 m+1}=(n+1) ! \sum_{\ell=0}^{m-1}(-1)^{m-1-\ell} b_{m, \ell} n^{\ell}, \quad n \geq 2, \quad 1 \leq m \leq \frac{1}{2}\left[n-\frac{1-(-1)^{n}}{2}\right]$

for derivative polynomials $P_{n}(x)$ of the tangent function $\tan x$, where $b_{m, \ell}$ is a sequence to be determined.

In the final section of this paper, we give a consequence of Theorem 1 and recommend some papers closely related to derivative polynomials of other elementary and applicable functions.

\section{Proof of Theorem 1}

Now we start out to simply prove our Theorems 1 as follows. 
In [36, Theorem 2.1] and [36, Corollaries 2.1 and 2.2], it was obtained that

$$
\begin{aligned}
& (\tan x)^{(n)}=(-i)^{n+1} \sum_{k=1}^{n+1} 2^{n+1-k}(k-1) ! S(n+1, k)(i \tan x-1)^{k}, \\
& (\tan x)^{(n)}=(\tan x+i) \sum_{k=1}^{n}(2 i)^{n-k} k ! S(n, k)(\tan x-i)^{k},
\end{aligned}
$$

and

$$
\begin{aligned}
(\tan x)^{(n)}=\sum_{k=0}^{n+1} & {\left[(-1)^{k+1} \cos \left(\frac{n+1+k}{2} \pi\right)\right.} \\
& \left.\times \sum_{\ell=\max \{1, k\}}^{n+1}(-1)^{n-\ell} 2^{n-\ell+1}(\ell-1) ! S(n+1, \ell)\left(\begin{array}{l}
\ell \\
k
\end{array}\right)\right] \tan ^{k} x .
\end{aligned}
$$

The identity (5) can be reformulated as

$$
\begin{gathered}
(\tan x)^{(n)}=-\cos \left(\frac{n+1}{2} \pi\right) \sum_{\ell=1}^{n+1}(-1)^{n-\ell} 2^{n-\ell+1}(\ell-1) ! S(n+1, \ell) \\
+\sum_{k=1}^{n+1}\left[(-1)^{k+1} \cos \left(\frac{n+1+k}{2} \pi\right) \sum_{\ell=k}^{n+1}(-1)^{n-\ell} 2^{n-\ell+1}(\ell-1) ! S(n+1, \ell)\left(\begin{array}{l}
\ell \\
k
\end{array}\right)\right] \tan ^{k} x .
\end{gathered}
$$

Consequently, we arrives at

$$
\begin{aligned}
a_{2 m-1,0} & =-\cos \left(\frac{2 m}{2} \pi\right) \sum_{\ell=1}^{2 m}(-1)^{2 m-\ell-1} 2^{2 m-\ell}(\ell-1) ! S(2 m, \ell) \\
& =(-1)^{m} \sum_{\ell=1}^{2 m}(-1)^{\ell} 2^{2 m-\ell}(\ell-1) ! S(2 m, \ell)
\end{aligned}
$$

for $m \geq 1$ and

$$
\begin{aligned}
a_{n, n+1-2 m}= & (-1)^{n} \cos ((n+1-m) \pi) \\
& \sum_{\ell=n+1-2 m}^{n+1}(-1)^{n-\ell} 2^{n-\ell+1}(\ell-1) ! S(n+1, \ell)\left(\begin{array}{c}
\ell \\
n+1-2 m
\end{array}\right) \\
= & (-1)^{m-1} \sum_{\ell=n+1-2 m}^{n+1}(-1)^{n-\ell} 2^{n+1-\ell}(\ell-1) ! S(n+1, \ell)\left(\begin{array}{c}
\ell \\
n+1-2 m
\end{array}\right)
\end{aligned}
$$

for $0 \leq m \leq \frac{1}{2}\left[n-\frac{1-(-1)^{n}}{2}\right]$. The proof of Theorem 1 is thus complete. 


\section{An open problem}

Now we would like to propose an open problem as follows.

The equation (2) means that

$$
(\tan x)^{(n)}=\sum_{k=0}^{\frac{1}{2}\left[n+\frac{1-(-1)^{n}}{2^{2}}\right]} a_{n, n-2 k+1} \tan ^{n-2 k+1} x .
$$

Differentiating with respect to $x$ on both sides of (6) gives

$$
\begin{gathered}
(\tan x)^{(n+1)}=\sum_{k=0}^{\frac{1}{2}\left[n+\frac{1-(-1)^{n}}{2}\right]} a_{n, n-2 k+1}(n-2 k+1) \tan ^{n-2 k} x\left(1+\tan ^{2} x\right) \\
=\sum_{k=0}^{\frac{1}{2}\left[n+\frac{1-(-1)^{n}}{2}\right]} a_{n, n-2 k+1}(n-2 k+1) \tan ^{n-2 k} x \\
+\sum_{k=0}^{\frac{1}{2}\left[n+\frac{1-(-1)^{n}}{2}\right]} a_{n, n-2 k+1}(n-2 k+1) \tan ^{n-2 k+2} x \\
=\sum_{k=1}^{\frac{1}{2}\left[n+\frac{1-(-1)^{n}}{2}\right]+1} a_{n, n-2 k+3}(n-2 k+3) \tan ^{n-2 k+2} x \\
+\sum_{k=0}^{\frac{1}{2}\left[n+\frac{1-(-1)^{n}}{2}\right]} a_{n, n-2 k+1}(n-2 k+1) \tan ^{n-2 k+2} x \\
\sum_{k=1}^{\frac{1}{2}\left[n+\frac{1-(-1)^{n}}{2}\right]}\left[a_{n, n-2 k+3}(n-2 k+3)+a_{n, n-2 k+1}(n-2 k+1)\right] \tan ^{n-2 k+2} x \\
+a_{n, n+1}(n+1) \tan ^{n+2} x+a_{n, \frac{1+(-1)^{n}}{2}} \frac{1+(-1)^{n}}{2} \tan ^{\frac{(-1)^{n}-1}{2}} x .
\end{gathered}
$$

Comparing this with

$$
(\tan x)^{(n+1)}=\sum_{k=0}^{\frac{1}{2}\left[n+1+\frac{1+(-1)^{n}}{2}\right]} a_{n+1, n-2 k+2(\tan x)^{n-2 k+2}}
$$


yields

$$
\begin{gathered}
a_{n+1, n+2}=a_{n, n+1}(n+1), \\
a_{n+1, \frac{1-(-1)^{n}}{2}} \tan \frac{1-(-1)^{n}}{2} x=a_{n, \frac{1+(-1)^{n}}{2}} \frac{1+(-1)^{n}}{2} \tan ^{\frac{(-1)^{n}-1}{2}} x,
\end{gathered}
$$

and

$$
a_{n+1, n-2 k+2}=a_{n, n-2 k+3}(n-2 k+3)+a_{n, n-2 k+1}(n-2 k+1)
$$

for $n \geq 1$ and $1 \leq k \leq \frac{1}{2}\left[n+\frac{1-(-1)^{n}}{2}\right]$.

The derivatives of the tangent function $\tan x$ in (1) means that $a_{0,1}=1$, $a_{1,2}=1, a_{2,3}=2$, and $a_{3,4}=2 \cdot 3$. Combining these values with (7) reveals that $a_{n, n+1}=n$ ! for all $n \geq 0$.

The derivatives of the tangent function $\tan x$ in (1) also means that $a_{1,0}=1$, $a_{2,1}=2$, and $a_{3,0}=2$. When $n=2 \ell$ for $\ell \geq 0$, the recurrence relation (8) becomes

$$
a_{2 \ell+1,0}=a_{2 \ell, 1} .
$$

When $k=1$, the recurrence relation (9) can be simplified as

$$
a_{n+1, n}=a_{n, n+1}(n+1)+a_{n, n-1}(n-1)=a_{n, n-1}(n-1)+(n+1) !
$$

for $n \geq 2$. From this recurrence relation, we acquire

$$
a_{n, n-1}=\frac{1}{3}(n+1) !, \quad n \geq 2 .
$$

When $k=2$, by (10), the recurrence relation (9) can be rearranged as

$$
a_{n+1, n-2}=a_{n, n-1}(n-1)+a_{n, n-3}(n-3)=a_{n, n-3}(n-3)+(n-1) \frac{(n+1) !}{3}
$$

for $n \geq 4$. Accordingly, it follows that

$$
a_{n, n-3}=\frac{5 n-8}{90}(n+1) !, \quad n \geq 4 .
$$

When $k=3$, by (11), the recurrence relation (9) can be rewritten as $a_{n+1, n-4}=a_{n, n-3}(n-3)+a_{n, n-5}(n-5)=a_{n, n-5}(n-5)+(n-3) \frac{5 n-8}{90}(n+1) !$ for $n \geq 6$. Therefore, it follows that

$$
a_{n, n-5}=\frac{35 n^{2}-203 n+264}{5670}(n+1) !, \quad n \geq 6 .
$$


Similarly as above processing, we can procure that

$$
\begin{gathered}
a_{n, n-7}=\frac{175 n^{3}-2205 n^{2}+8654 n-10272}{340200}(n+1) !, \quad n \geq 8, \quad \\
a_{n, n-9}=\frac{385 n^{4}-8470 n^{3}+66539 n^{2}-217910 n+244704}{11226600}(n+1) !, \quad n \geq 10,
\end{gathered}
$$

and the like. Accordingly, from (10), (11), (12), (13), and (14), we can conclude that

$$
\begin{aligned}
& a_{n, n-2 m+1}=(n+1) ! \sum_{\ell=0}^{m-1}(-1)^{m-1-\ell} b_{m, \ell} n^{\ell}, \\
& n \geq 2, \quad 1 \leq m \leq \frac{1}{2}\left[n-\frac{1-(-1)^{n}}{2}\right] .
\end{aligned}
$$

Substituting this conclusion into (9) leads to

$$
\begin{gathered}
(n+2) ! \sum_{\ell=0}^{k-1}(-1)^{k-1-\ell} b_{k, \ell}(n+1)^{\ell}=(n-2 k+3)(n+1) ! \sum_{\ell=0}^{k-2}(-1)^{k-2-\ell} b_{k-1, \ell} n^{\ell} \\
+(n-2 k+1)(n+1) ! \sum_{\ell=0}^{k-1}(-1)^{k-1-\ell} b_{k, \ell} n^{\ell} \\
\sum_{\ell=0}^{k-1}(-1)^{\ell+1}\left[(n+2)(n+1)^{\ell}-(n-2 k+1) n^{\ell}\right] b_{k, \ell} \\
=(n-2 k+3) \sum_{\ell=0}^{k-2}(-1)^{\ell} n^{\ell} b_{k-1, \ell},
\end{gathered}
$$

where $n \geq 4$ and $2 \leq k \leq \frac{1}{2}\left[n-\frac{1-(-1)^{n}}{2}\right]$. Note that the sequence $b_{k, \ell}$ are independent of $n$.

To the best of our knowledge, we think that it is much difficult to explicitly determine the sequence $b_{m, \ell}$ in (15). Can one present a closed form for the sequence $b_{m, \ell}$ in $(15)$ ?

\section{Remarks}

Finally we comment on Theorem 1 and recommend some references closely related to derivative polynomials of other elementary and applicable functions. 
Remark 1 The expression (3) implies an explicit formula

$$
\mathrm{T}_{2 \mathrm{~m}-1}=(-1)^{\mathrm{m}} \sum_{\ell=1}^{2 \mathrm{~m}}(-1)^{\ell} 2^{2 \mathrm{~m}-\ell}(\ell-1) ! \mathrm{S}(2 \mathrm{~m}, \ell), \quad \mathrm{m} \geq 1
$$

for tangent numbers $\mathrm{T}_{2 \mathrm{~m}-1}$ which can be generated by

$$
\tan x=\sum_{k=1}^{\infty} T_{2 k-1} \frac{x^{2 k-1}}{(2 k-1) !}, \quad|x|<\frac{\pi}{2} .
$$

For more information on tangent numbers $\mathrm{T}_{2 \mathrm{~m}-1}$, please refer to $[1,11,14,36]$ and the closely related references therein.

Remark 2 It is worthwhile to recommending the paper [2] which was found on 3 March 2017 by the authors.

Remark 3 Except the above-mentioned literature, there are other papers such as $[6,7,13,15,16,17,18,19,22,23,24,25,28,29,31,34,35,36,37]$ and the closely related references therein to discuss derivative polynomials of other elementary and applicable functions.

\section{Acknowledgements}

The authors are grateful to the anonymous referees for their careful corrections to the original version of this paper.

\section{References}

[1] L. C. Bouvier, Analise transcendante. Loi du développement de la tangente en fonction de l'arc, Ann. Math. Pures Appl. [Ann. Gergonne], 14 (1823/24), 347-349; Available online at http://www.numdam.org/ numdam-bin/fitem?id=AMPA_1823-1824__14__347_1. (French)

[2] K. N. Boyadzhiev, Derivative polynomials for tanh, tan, sech and sec in explicit form, Fibonacci Quart., 45 (2007), no. 4, 291-303 (2008); Available online at https://arxiv.org/abs/0903.0117.

[3] C.-P. Chen, F. Qi, A double inequality for remainder of power series of tangent function, Tamkang J. Math., 34 (2003), no. 4, 351-355; Available online at http://dx.doi.org/10.5556/j.tkjm.34.2003.236. 
[4] C.-P. Chen, F. Qi, A double inequality for remainder of power series of tangent function, RGMIA Res. Rep. Coll., 5 (2002), Suppl., Art. 2; Available online at http://rgmia.org/v5(E).php.

[5] B.-N. Guo, Q.-M. Luo, F. Qi, Sharpening and generalizations of Shafer-Fink's double inequality for the arc sine function, Filomat, 27 (2013), no. 2, 261-265; Available online at http://dx.doi .org/10.2298/ FIL1302261G.

[6] B.-N. Guo, F. Qi, Explicit formulae for computing Euler polynomials in terms of Stirling numbers of the second kind, J. Comput. Appl. Math., 272 (2014), 251-257; Available online at http://dx.doi.org/10.1016/ j.cam.2014.05.018.

[7] B.-N. Guo, F. Qi, Some identities and an explicit formula for Bernoulli and Stirling numbers, J. Comput. Appl. Math., 255 (2014), 568-579; Available online at http://dx.doi.org/10.1016/j.cam.2013.06.020.

[8] M. E. Hoffman, Derivative polynomials for tangent and secant, Amer. Math. Monthly, 102 (1995), no. 1, 23-30; Available online at http://dx. doi.org/10.2307/2974853.

[9] M. E. Hoffman, Derivative polynomials, Euler polynomials, and associated integer sequences, Electron. J. Combin, 6 (1999), Research Paper 21, 13 pages; Available online at http://www.combinatorics.org/Volume_ 6/Abstracts/v6i1r21.html.

[10] R. K. Morley, Brief notes and comments. Successive derivatives of $\tan x$, National Mathematics Magazine, 19 (1945), no. 6, 311-312; Available online at http://www.jstor.org/stable/3030048.

[11] F. Qi, Derivatives of tangent function and tangent numbers, Appl. Math. Comput., 268 (2015), 844-858; Available online at http://dx.doi.org/ $10.1016 /$ j . amc. 2015.06.123.

[12] F. Qi, Explicit formulas for the $\mathrm{n}$-th derivatives of the tangent and cotangent functions, arXiv preprint, (2012), available online at http: //arxiv.org/abs/1202.1205.

[13] F. Qi, V. Čerňanová, and Y. S. Semenov, On tridiagonal determinants and the Cauchy product of central Delannoy numbers, ResearchGate Working Paper, (2016), available online at http://dx.doi.org/10.13140/RG.2. 1.3772 .6967$. 
[14] F. Qi, J. Gélinas, Revisiting Bouvier's paper on tangent numbers, Adv. Appl. Math. Sci., 16 (2017), no. 8, 275-281.

[15] F. Qi, Explicit formulas for the convolved Fibonacci numbers, ResearchGate Working Paper, (2016), available online at http://dx.doi.org/10. 13140/RG.2.2.36768.17927.

[16] F. Qi, B.-N. Guo, Explicit formulas and recurrence relations for higher order Eulerian polynomials, Indag. Math., 28 (2017), no. 4, 884-891; Available online at https://doi.org/10.1016/j.indag.2017.06.010.

[17] F. Qi and B.-N. Guo, Explicit formulas for derangement numbers and their generating function, J. Nonlinear Funct. Anal., 2016, Article ID 45, 10 pages.

[18] F. Qi, Q. Zou, and B.-N. Guo, Some identities and a matrix inverse related to the Chebyshev polynomials of the second kind and the Catalan numbers, Preprints, 2017, 2017030209, 25 pages; Available online at https://doi.org/10.20944/preprints201703.0209.v2.

[19] F. Qi and B.-N. Guo, Several explicit and recursive formulas for the generalized Motzkin numbers, Preprints, 2017, 2017030200, 11 pages; Available online at http://dx.doi.org/10.20944/preprints201703.0200. v1.

[20] F. Qi, B.-N. Guo, Sharpening and generalizations of Shafer's inequality for the arc sine function, Integral Transforms Spec. Funct., 23 (2012), no. 2, 129-134; Available online at http://dx.doi.org/10.1080/10652469. 2011.564578 .

[21] F. Qi, B.-N. Guo, Sharpening and generalizations of Shafer's inequality for the arc tangent function, arXiv preprint, (2009), available online at http://arxiv.org/abs/0902.3298.

[22] F. Qi, B.-N. Guo, A diagonal recurrence relation for the Stirling numbers of the first kind, Appl. Anal. Discrete Math., 12 (2018), no. 1, in press; Available online at https://doi.org/10.2298/AADM170405004Q.

[23] F. Qi, B.-N. Guo, Some properties of the Hermite polynomials and their squares and generating functions, Preprints, 2016, 2016110145, 14 pages; Available online at http://dx.doi.org/10.20944/preprints201611. 0145.v1. 
[24] F. Qi, B.-N. Guo, Viewing some nonlinear ODEs and their solutions from the angle of derivative polynomials, ResearchGate Working Paper, (2016), available online at http://dx.doi.org/10.13140/RG.2.1.4593.1285.

[25] F. Qi, B.-N. Guo, Viewing some ordinary differential equations from the angle of derivative polynomials, Preprints, 2016, 2016100043, 12 pages; Available online at http://dx.doi.org/10.20944/preprints201610. 0043.v1.

[26] F. Qi, D.-W. Niu, B.-N. Guo, Refinements, generalizations, and applications of Jordan's inequality and related problems, J. Inequal. Appl., 2009 (2009), Article ID 271923, 52 pages; Available online at http: //dx.doi.org/10.1155/2009/271923.

[27] F. Qi, X.-T. Shi, F.-F. Liu, D. V. Kruchinin, Several formulas for special values of the Bell polynomials of the second kind and applications, $J$. Appl. Anal. Comput., 7 (2017), no. 3, 857-871; Available online at https: //doi.org/10.11948/2017054.

[28] F. Qi, J.-L. Wang, B.-N. Guo, Notes on a family of inhomogeneous linear ordinary differential equations, Adv. Appl. Math. Sci., 17 (2018), no. 4, $361-368$.

[29] F. Qi, J.-L. Wang, B.-N. Guo, Simplifying and finding nonlinear ordinary differential equations, ResearchGate Working Paper, (2017), available online at https://doi.org/10.13140/RG.2.2.28855.32166.

[30] F. Qi, S.-Q. Zhang, B.-N. Guo, Sharpening and generalizations of Shafer's inequality for the arc tangent function, J. Inequal. Appl., 2009, Article ID 930294, 9 pages; Available online at http://dx.doi.org/10.1155/ $2009 / 930294$.

[31] F. Qi, J.-L. Zhao, Some properties of the Bernoulli numbers of the second kind and their generating function, ResearchGate Working Paper (2017), available online at http://dx.doi.org/10.13140/RG.2.2. 13058.27848.

[32] F. Qi, M.-M. Zheng, Explicit expressions for a family of the Bell polynomials and applications, Appl. Math. Comput., 258 (2015), 597-607; Available online at http://dx.doi.org/10.1016/j.amc.2015.02.027.

[33] J.-L. Zhao, Q.-M. Luo, B.-N. Guo, F. Qi, Remarks on inequalities for the tangent function, Hacettepe J. Math. Statist., 41 (2012), no. 4, 499-506. 
[34] J.-L. Zhao, F. Qi, Two explicit formulas for the generalized Motzkin numbers, J. Inequal. Appl., 2017, 2017:44, 8 pages; Available online at http://dx.doi.org/10.1186/s13660-017-1313-3.

[35] J.-L. Zhao, J.-L. Wang, F. Qi, Derivative polynomials of a function related to the Apostol-Euler and Frobenius-Euler numbers, J. Nonlinear Sci. Appl., 10 (2017), no. 4, 1345-1349; Available online at http://dx.doi. org/10.22436/jnsa.010.04.06.

[36] A.-M. Xu, G.-D. Cen, Closed formulas for computing higher-order derivatives of functions involving exponential functions, Appl. Math. Comput., 270 (2015), 136-141; Available online at http://dx.doi.org/10.1016/ j.amc.2015.08.051.

[37] A.-M. Xu, Z.-D. Cen, Some identities involving exponential functions and Stirling numbers and applications, J. Comput. Appl. Math., 260 (2014), 201-207; Available online at http://dx.doi.org/10.1016/j.cam. 2013. 09.077. 